\title{
Venous Tumor Thrombosis and Pulmonary Metastases in a Case of Adrenal Neuroblastoma- Occurrence of Two Rare Manifestations in a Single Patient: Case Report and Review of Literature
}

\author{
Somesh Singh ${ }^{1}$ Seema Kembhavi ${ }^{1} \quad$ Sneha Shah ${ }^{2} \quad$ Tushar Vora $^{3} \quad$ Girish Chinnaswamy ${ }^{3}$ Sajid Qureshi ${ }^{4}$ \\ ${ }^{1}$ Department of Radiodiagnosis, Tata Memorial Centre, \\ Mumbai, India \\ 2Department of Nuclear Medicine, Tata Memorial Centre, \\ Address for correspondence Somesh Singh, MD, Department \\ of Radiodiagnosis, Tata Memorial Centre, Mumbai 400012, \\ Maharashtra, India (e-mail: someshsingh88@gmail.com).
} Mumbai, India

${ }^{3}$ Department of Paediatric Oncology, Tata Memorial Centre, Mumbai, India

${ }^{4}$ Department of Paediatric Surgical Oncology, Tata Memorial Centre, Mumbai, India

J Gastrointestinal Abdominal Radiol ISGAR:2020;3(suppl S1):S49-S53

\begin{abstract}
Keywords

- neuroblastoma

- pulmonary metastases

- tumor thrombosis

Unlike other pediatric extra cranial solid neoplasms, neuroblastomas have very low incidence of pulmonary metastases. Neuroblastomas also tend to encase rather than infiltrate the major vessels with very low incidence of tumor thrombus. Even in cases with inferior vena cava tumor thrombosis, pulmonary metastases are a rare occurrence. These patients usually show widespread disseminated disease at presentation. We present a rare case of adrenal neuroblastoma with tumor thrombus and bilateral pulmonary metastases at presentation. This presentation can be confused with adrenocortical carcinoma and differentiating between the two is essential for further management.
\end{abstract}

\section{Case Presentation}

A 4-year-old girl presented with pain abdomen for 4 months and a lumpy feeling in left upper abdomen for 2 months. On physical examination, a vague mass was palpable at the left hypochondriac and lumbar regions. The mass was nontender, firm in consistency, and was not ballotable. A few prominent abdominal veins were also seen. Blood workup revealed elevated serum lactate dehydrogenases levels (763 IU, normal range: 120-220 IU). The adrenal cortical hormonal levels were within normal limits. Early morning serum cortisol level was $80 \mathrm{ng} / \mathrm{mL}$ (normal less than $200 \mathrm{ng} / \mathrm{mL}$ ). The serum catecholamine levels were also within normal limits. Serum normetanephrine was $4 \mathrm{pg} / \mathrm{mL}$ (normal $<100 \mathrm{pg} / \mathrm{mL}$ ) and serum metanephrine was $2 \mathrm{pg} / \mathrm{mL}$ (normal $<60 \mathrm{pg} / \mathrm{mL}$ ). The hemogram was also within normal limits.

\section{Investigations and Imaging Findings}

\section{Contrast-Enhanced Computed Tomography of} Abdomen and Pelvis

A contrast-enhanced computed tomography (CT) scan of abdomen and pelvis was performed on a 16-slice multidetector CT scanner (Siemens Somatom Sensation 16, Seimens Healthineers, Erlangen, Germany) after administration of $40 \mathrm{~mL}$ intravenous nonionic contrast medium with automated exposure control. Contrast medium used was iohexol of strength $300 \mathrm{mg} / \mathrm{mL}$ (Omnipaque, GE Healthcare, Marlborough, Massachusetts, United States). The CT showed a large heterogeneously and mildly enhancing left suprarenal mass (as shown in - Fig. 1) with coarse calcifications within. The mass was indenting the spleen and the left kidney was displaced in inferior direction. No definite
DOI https://doi.org/ 10.1055/s-0039-3402611 ISSN 2581-9933. (c) 2020. Indian Society of Gastrointestinal and Abdominal Radiology. This is an open access article published by Thieme under the terms of the Creative Commons Attribution-NonDerivative-NonCommercial-License, permitting copying and reproduction so long as the original work is given appropriate credit. Contents may not be used for commercial purposes, or adapted, remixed, transformed or built upon. (https://creativecommons.org/licenses/by-nc-nd/4.0/)

Thieme Medical and Scientific Publishers Pvt. Ltd., A-12, 2nd Floor, Sector 2, Noida-201301 UP, India 
renal or splenic infiltration was seen. In addition, multiple conglomerate and discrete retroperitoneal nodes were seen, encasing the abdominal aorta. Multiple bilobar hypodense hepatic lesions, suggestive of metastases were also seen (-Fig. 2). In addition, a large filling defect was seen involving the left renal vein, extending into the inferior vena cava (IVC), reaching up to the retrohepatic IVC, up to the level of hepatic venous confluence. It showed similar morphology and enhancement pattern as the left suprarenal mass and was causing expansion of the involved vessels, suggestive of tumor thrombus. Basal cuts of thorax showed multiple nodules in bilateral lower pulmonary lobes, suggestive of metastases (-Fig. 2).

\section{Histopathology}

Biopsy of the left suprarenal mass was performed under ultrasound guidance which showed stroma poor poorly differentiated neuroblastoma with low Mitosis-Karyorrhexis index (MKI). Histology was of unfavorable category as per the International Neuroblastoma Pathology Classification system. ${ }^{1}$ Further, evaluation with immunohistochemistry showed the tumor to be positive for synaptophysin. Molecular studies showed N-MYC amplification. As per staging requirement for neuroblastoma, bilateral bone marrow biopsies were done which showed normocellular uninvolved marrow.

\section{8-Fluorodeoxyglucose Positron Emission Tomography-Computed Tomography}

A further staging work-up with fluorodeoxyglucose positron emission tomography-computed tomography (FDG PET-CT) was done. MIBG (metaiodobenzylguanidine) scan was not performed after discussion at multidisciplinary meet as the tumor was poorly differentiated. PET-CT showed hypermetabolic left suprarenal mass with hypermetabolic tumor thrombus within the left renal vein and IVC, as well as multiple metastatic hypermetabolic retroperitoneal nodes, and bilobar hepatic metastases. In addition, multiple metastatic and hypermetabolic marrow lesions were seen ( - Fig. 3). Multiple bilateral pulmonary metastases were also seen distributed randomly in both lungs with a basal predominance ( - Fig. 2 ).

\section{Differential Diagnosis}

The main differential based on initial CT scan of abdomen was adrenocortical carcinoma as the tumor was arising from the suprarenal region. Adrenocortical carcinoma has a bimodal distribution of age with peaks in first and fourth decades. These can show coarse calcification in up to $33 \%$ of cases $^{2}$ and lungs are the most common sites of metastases. ${ }^{3}$ Intravascular tumor thrombosis can be found in up to $20 \%$ cases of adrenocortical carcinoma. ${ }^{4} \mathrm{Up}$ to $40 \%$ cases of adrenocortical carcinomas can be nonfunctional. ${ }^{5}$
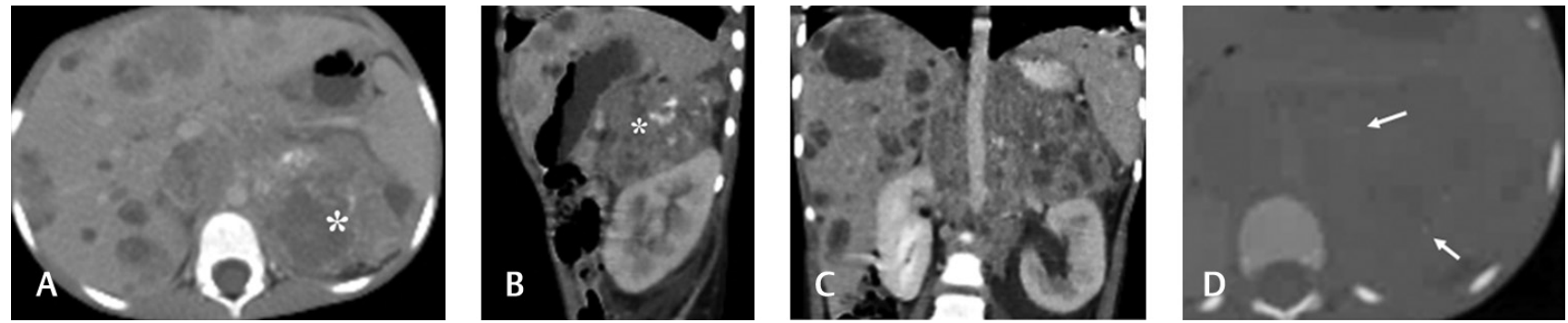

Fig. 1 Axial (A) and coronal (B) images show a large left suprarenal mass $\left(^{*}\right)$ showing heterogeneous enhancement and displacing the kidney inferiorly. The fat planes with spleen and kidney appear maintained. Coronal CT (C) shows multiple conglomerate and discrete retroperitoneal nodes, encasing the abdominal aorta. Bone window (D) demonstrates coarse calcifications within (shown by arrows). CT, computed tomography.
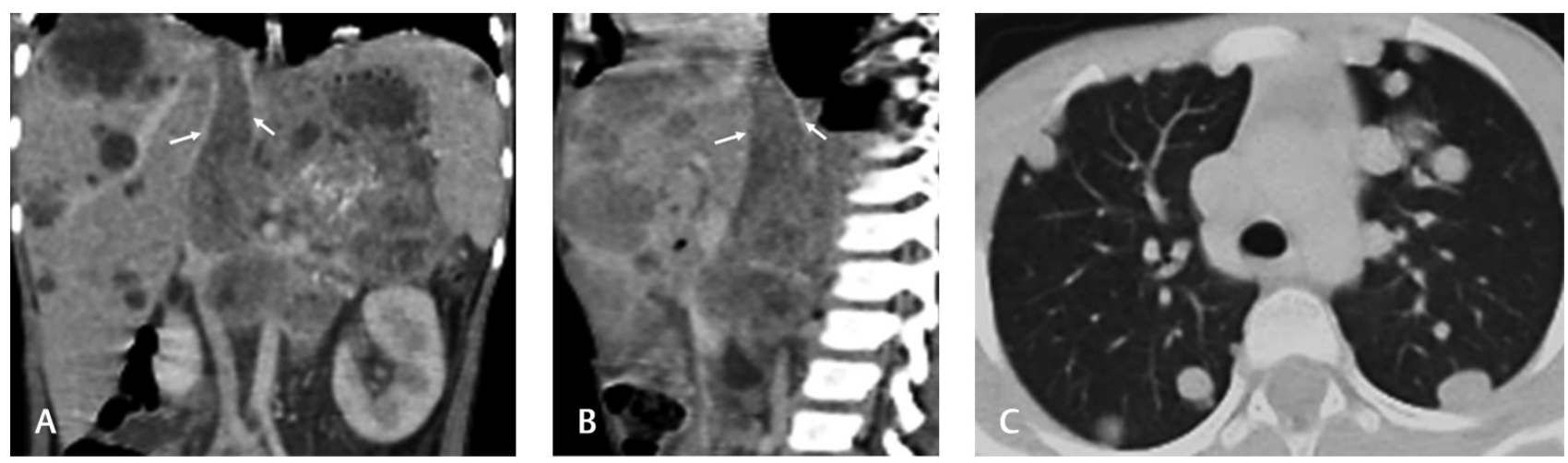

Fig. 2 Coronal (A) and Sagittal (B) images show multiple bilobar hepatic metastases. In addition, a large filling defect was seen involving the inferior vena cava (marked by arrows), reaching up to the retrohepatic IVC, up to the level of hepatic vein confluence. CT axial cut in lung window (C) shows multiple varying sized pulmonary nodules and masses, scattered randomly in both lungs with a basal predominance, suggestive of metastases. CT, computed tomography; IVC, inferior vena cava. 

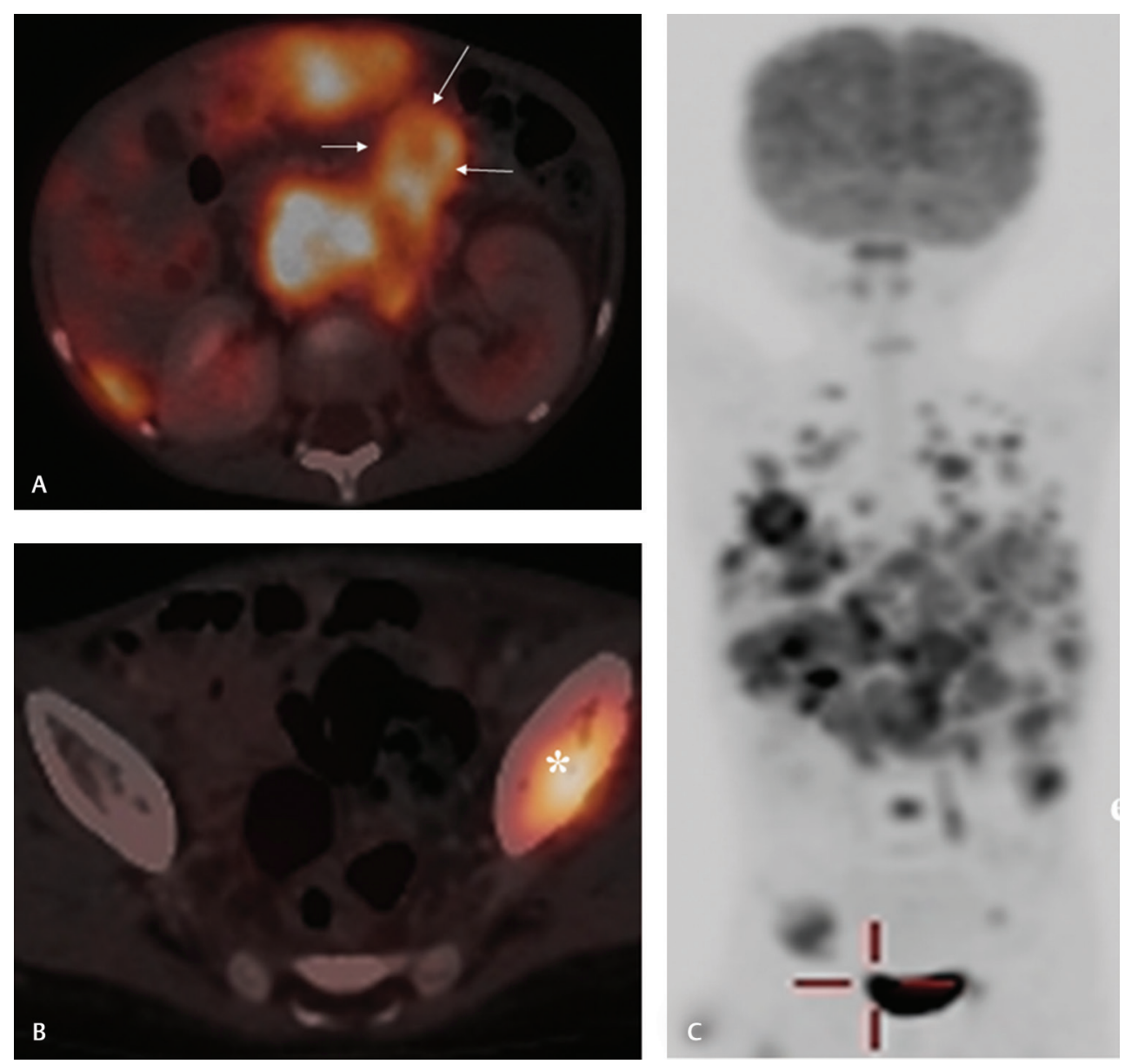

Fig. 3 FDG PET CT of the patient showed conglomerate hypermetabolic retroperitoneal nodes (arrows in A). It also shows metabolically active and inactive hepatic deposits. Hypermetabolic metastatic deposit involving left acetabulum is shown by * in B. Maximum intensity projection (MIP) images shows disseminated disease with multiple metabolically active nodules in both lungs (C). No FDG avid lesions were seen in brain. FDG PET-CT, fluorodeoxyglucose positron emission tomography-computed tomography.

A pediatric lumbar region mass with IVC thrombus points toward a possible Wilm's tumor. However, in our case, fat planes with left kidney were maintained throughout with the kidney displaced inferiorly. Hence, Wilm's tumor was not in our list of possible differentials.

\section{Treatment, Outcome, and Follow-up}

As the patient had high-risk features, she was put on rapid COJEC chemotherapy regimen (C, cisplatin; O, vincristine; J, carboplatin; E, etoposide; and C, cyclophosphamide). She was scheduled for postchemotherapy FDG PET-CT for reassessment for surgery. After the fifth cycle of chemotherapy, the child developed febrile neutropenia with tachycardia, tachypnoea, and hypotension. Subsequently, the patient developed cardiac arrest and unfortunately succumbed to same.

\section{Discussion}

Neuroblastoma spectrum comprises the most common extracranial solid neoplasm in pediatric population ${ }^{6}$ arising from neural crest cells and can show a wide range of clinical and imaging presentation. ${ }^{7}$ Bone and bone marrow metastases are the most common sites of metastases in neuroblastoma. ${ }^{8}$ Unlike other pediatric extra cranial neoplasms, where lungs are usually most common site of metastases, pulmonary metastases are very unusual in neuroblastoma even in stage $4 .{ }^{8,9,10}$ Stigall et al ${ }^{11}$ described the following three types of pulmonary involvement: (1) direct extension, (2) hematogenous spread, and (3) lymphatic spread. Our patient likely had hematogenous spread, as they were distributed randomly in both lungs with a basal predominance and also probably due to existing large volume tumor thrombus within the IVC.

A recent large retrospective study by International Neuroblastoma Risk Group (INRG) showed overall incidence of pulmonary metastases to be $3.6 \%{ }^{12}$ which further bolstered the current clinical practice of not performing chest imaging in suspected case of neuroblastoma. It has been postulated that this may be due hostile environment within the lungs, inhibiting the growth of distant hematogenous seedlings in neuroblastoma. ${ }^{11}$ Even in published case reports of neuroblastomas with tumor thrombus within the inferior vena cava, detection of pulmonary metastases was uncommon. ${ }^{13-15}$ A study found that neuroblastoma cells do not passively travel along the direction of blood flow. ${ }^{16}$ 
As it has been postulated that the pulmonary environment is relatively hostile for the growth of neuroblastoma metastases, histologically and biologically aggressive tumors tend to show higher incidence of pulmonary metastases. ${ }^{12}$ The pathological, serological, and molecular features of such unfavorable histology are poor differentiation, elevated lactate dehydrogenease (LDH) levels, and N-MYC amplification, respectively. ${ }^{12}$ All these findings were present in our study. The elevated LDH levels can reflect higher proliferation of the tumor with higher cell turnover or could be due to injury to the pulmonary tissue by the growing deposit. ${ }^{12}$ Our patient did not show any evidence of neuroparenchymal metastases. While the calvarium is a common site of neuroblastoma metastases, neuroparenchymal metastases are rare. ${ }^{8}$ Patients presenting with pulmonary metastases have been shown to have a higher incidence of neuroparenchymal metastases well..$^{2}$ It is important to note that INRG study showed that while the lung metastases are seen in association with disseminated disease to other site, these are not independent prognostic marker of a poor outcome.

In addition, our patient showed a large tumor thrombus extending from left renal vein into the IVC up to the hepatic venous confluence. Presence of IVC tumor thrombosis is a rare occurrence in neuroblastoma and is an imaging feature more commonly associated with Wilms' tumor with only few case reports published..$^{17}$ As with the case of pulmonary metastases, presence of tumor thrombosis in a case of neuroblastoma indicated an aggressive tumor and, hence, a poor prognosis and requiring intensive chemotherapy. ${ }^{18}$

\section{Conclusion}

In conclusion, this was a unique presentation of pediatric adrenal neuroblastoma with simultaneous presence of two rare manifestations, presence of pulmonary metastases and tumor thrombus within the IVC. Due to this atypical presentation, the tumor was suspected to be an adrenocortical carcinoma. Consistent with the literature, the presence of these rare features was associated with aggressive histology, elevated LDH, and N-MYC mutation.

\section{Learning Points}

- Pediatric neuroblastomas may show extensive hematogenous metastases but surprisingly have very low incidence of pulmonary metastases even in advanced disease. Also, these tumors usually encase and compress the adjacent vascular structures and very rarely cause infiltration of vessels with extension along them as a tumor thrombus.

- Presence of a tumor thrombus does not imply a higher chance of pulmonary metastasis as it has been postulated than pulmonary environment is not very conducive for growth of these seedlings. Hence, presence of these two features simultaneously makes this a rare presentation.

- Presence of either of these (tumor thrombus and pulmonary metastases) imply aggressive tumor as seen by unfavorable histology, elevated LDH levels, and N-MYC amplification in this case.

- Presence of pulmonary metastases is not an independent poor prognostic marker in these patients but these patients have a poor clinical outcome due to aggressive underlying tumor per se.

- A suprarenal mass with multiple bilateral pulmonary metastases in a pediatric patient should arouse the suspicion of an adrenocortical carcinoma which can also show coarse calcification within. A histopathological examination is hence essential as the management of the two tumors varies greatly.

\section{Conflict of Interest}

None declared.

\section{References}

1 Shimada H, Ambros IM, Dehner LP, et al. The International Neuroblastoma Pathology Classification (the Shimada system). Cancer 1999;86(2):364-372

2 Johnson PT, Horton KM, Fishman EK. Adrenal mass imaging with multidetector CT: pathologic conditions, pearls, and pitfalls. Radiographics 2009;29(5):1333-1351

3 Brennan MF. Adrenocortical carcinoma. CA Cancer J Clin 1987;37(6):348-365

4 Pereira RM, Michalkiewicz E, Sandrini F, et al. Tumores do córtex adrenal na infância. Arq Bras Endocrinol Metabol 2004;48(5):651-658

$5 \mathrm{Ng} \mathrm{L}$, Libertino JM. Adrenocortical carcinoma: diagnosis, evaluation and treatment. J Urol 2003;169(1):5-11

6 Matthay KK, Shulkin B, Ladenstein R, et al. Criteria for evaluation of disease extent by (123)I-metaiodobenzylguanidine scans in neuroblastoma: a report for the International Neuroblastoma Risk Group (INRG) task force. Br J Cancer 2010;102(9):1319-1326

7 Kembhavi SA, Shah S, Rangarajan V, Qureshi S, Popat P, Kurkure P. Imaging in neuroblastoma: an update. Indian J Radiol Imaging 2015;25(2):129-136

8 DuBois SG, Kalika Y, Lukens JN, et al. Metastatic sites in stage IV and IVS neuroblastoma correlate with age, tumor biology, and survival. J Pediatr Hematol Oncol 1999;21(3):181-189

9 Kammen BF, Matthay KK, Pacharn P, Gerbing R, Brasch RC, Gooding CA. Pulmonary metastases at diagnosis of neuroblastoma in pediatric patients: CT findings and prognosis. AJR Am J Roentgenol 2001;176(3):755-759

10 Cowie F, Corbett R, Pinkerton CR. Lung involvement in neuroblastoma: incidence and characteristics. Med Pediatr Oncol 1997;28(6):429-432

11 Stigall R, Smith WL, Franken EA, Smith JA, Crussi FG. [Intrapulmonic metastatic neuroblastoma]. Ann Radiol (Paris) 1979;22(2,3):223-227

12 Dubois SG, London WB, Zhang Y, et al. Lung metastases in neuroblastoma at initial diagnosis: A report from the International Neuroblastoma Risk Group (INRG) project. Pediatr Blood Cancer 2008;51(5):589-592

13 Bagatell R, Morgan E, Cosentino C, Whitesell L. Two cases of pediatric neuroblastoma with tumor thrombus in the inferior vena cava. J Pediatr Hematol Oncol 2002;24(5):397-400

14 Custodio CM, Semelka RC, Balci NC, Mitchell KM, Freeman JA. Adrenal neuroblastoma in an adult with tumor thrombus in the inferior vena cava. J Magn Reson Imaging 1999;9(4):621-623

15 Nagda SN, Lo SS, Melian E, Manera R, Emami B. Unusual thoracic problems in patients with malignancies: case 1 . 
Neuroblastoma presenting with intracardiac tumor thrombus. J Clin Oncol 2005;23(12):2856-2857

16 de la Monte SM, Moore GW, Hutchins GM. Nonrandom distribution of metastases in neuroblastic tumors. Cancer 1983;52(5):915-925

17 Shuichiro U, Yuichi T, Hisao Y, Yoshiko H, Takaharu O, Noriaki U. Abdominal neuroblastoma with inferior vena cava and right atrial extension. J Pediatr Surg Case Rep 2013;1(6):119-121

18 Gaetan G, Ouimet A, Lapierre C, Teira P, Sartelet H. Neuroblastoma presenting like a Wilms' tumor with thrombus in inferior vena cava and pulmonary metastases: a case series. Springerplus 2014;3:351 